\title{
Atomic Order and the Interaction of Electronic and Magnetic Subsystems in Epitaxial LaSr(Ca)MnO Films
}

\author{
V.D. OKuneV ${ }^{a}$, Z.A. SAmoilenko ${ }^{a}$, T.A. D'YaChenko ${ }^{a}$, \\ A. ABAl'osheV ${ }^{b}$, M. Baran ${ }^{b}$, P. GierŁoWski ${ }^{b}$, \\ S.J. LeWANDOWSKI ${ }^{b}$, A. SzEWCZYK ${ }^{b}$ AND R. SzYMCZAK ${ }^{b}$ \\ ${ }^{a}$ A. Galkin Donetsk Institute for Physics \& Technology \\ National Academy of Sciences of Ukraine, Donetsk \\ ${ }^{b}$ Institute of Physics, Polish Academy of Sciences \\ al. Lotników 32/46, 02-668 Warszawa, Poland
}

\begin{abstract}
We have investigated the structure, and electric, optical, and magnetic properties of $\mathrm{LaSr}(\mathrm{Ca}) \mathrm{MnO}$ films containing atomic clusters of various types coherently built into the basic crystallographic matrix. Below the transition to the metallic state, the electrical conductivity of the films is determined by tunneling of charge carriers between metallic clusters. We have found that for each sample there exists a threshold value of magnetic ordering, above which the magnetic subsystem starts to affect actively the film conductivity. The observed increase of conductivity with decreasing temperature is caused by the fact that in the process of magnetic ordering of the samples the cluster size and concentration of metallic phase increase. Experimental results are in agreement with theoretical calculations.
\end{abstract}

PACS numbers: $75.30 . \mathrm{Kz}, 75.47 . \mathrm{Lx}, 61.50 . \mathrm{Ks}$

\section{Introduction}

One of the important integral characteristics, reflecting the effects of interaction between electronic and magnetic subsystems in manganites, is the dependence of sample resistance $R$ on temperature $T$. Its behavior is dominated by the appearance of a maximum on the $R(T)$ curve, below which the resistance drops with decreasing temperature [1-7]. Individual samples may differ not only by the 
position of the maximum on $R(T)$ curves, but also by the rate $\mathrm{d} R / \mathrm{d} T$ of resistance changes. Monotonic decrease of resistance usually extends over an interval $200-300^{\circ} \mathrm{C}$ wide, but there are known cases of more rapid $R(T)$ changes concentrated in a $20-30^{\circ} \mathrm{C}$ interval with the $(1 / R) \mathrm{d} R / \mathrm{d} T$ derivative approaching $3-4 \% / \mathrm{K}$ [4-6]. Assuming that the reason of the observed differences in sample behavior is in the interaction of the atomic, electronic, and magnetic subsystems, we have investigated the details of this mechanism.

\section{Experimental}

The investigated samples were obtained by pulsed laser deposition ( KrF laser, $\tau=25 \mathrm{~ns}[8], \Phi=1.5-2.5 \mathrm{~J} / \mathrm{cm}^{2}$ on the target surface, $\mathrm{La}_{0.6} \mathrm{Sr}_{0.2} \mathrm{Mn}_{1.2} \mathrm{O}_{3}$ and $\mathrm{La}_{2 / 3} \mathrm{Ca}_{1 / 3} \mathrm{MnO}_{3}$ targets) onto various substrates at deposition temperatures $T_{\mathrm{s}}$ varying between $650^{\circ} \mathrm{C}$ and $730^{\circ} \mathrm{C}$. The resultant samples exhibited an orthorhombic structure. Transport measurements in the $4.2-300 \mathrm{~K}$ temperature range were made using the conventional four-probe technique. Magnetization of the samples was measured in a SQUID magnetometer. Structural investigation was carried out using the longwave $\mathrm{Cr} K_{\alpha} \mathrm{X}$-ray radiation.

\section{Results and discussion}

In the majority of the investigated samples, the $R(T)$ dependence could be considered as indicating metallic conductivity, in the sense that the formal $\mathrm{d} R / \mathrm{d} T>0$ criterion was satisfied. However, the comparison of the measured $\sigma$ values to the minimal metallic conductivity [10]

$$
\sigma_{\min }=\frac{\pi e^{2}}{4 z \hbar a}\left(\frac{B}{V_{0}}\right)_{\text {crit }}^{2},
$$

where $z$ is the coordination number and $B$ is the width of the band, yields $\sigma \ll \sigma_{\min }$. Indeed, for $\mathrm{LaCa}(\mathrm{Sr}) \mathrm{MnO} \sigma_{\min }=100 \div 1000 \Omega^{-1} \mathrm{~cm}^{-1}$, while the $R(T)$ characteristics reveal maxima of resistivity even at $\sigma=0.6-1.5 \Omega^{-1} \mathrm{~cm}^{-1}$, i.e. $2-3$ orders of magnitude below $\sigma_{\text {min }}$. For the metallic phase concentration $C_{m}<0.5$ (i.e. below the percolation threshold for films [11]), the samples apparently are in the dielectric state and their electric conductivity is determined by tunneling of charge carriers between mesoscopic regions (grains, clusters) with metallic conductivity. Indeed, X-ray structural investigation (Fig. 1) shows mesoscopically ordered clusters of a nearly constant size fluctuating around $100 \AA$, coherently built into the basic matrix of long range order. Among the clusters, the most important role is played by those, which are composed of $\mathrm{Mn}-\mathrm{O}$ planes, determining the transport and magnetic properties of $\mathrm{LaSr}(\mathrm{Ca}) \mathrm{MnO}$. Assuming that these grains have the size $D$ and are arranged in a regular matrix, the sample resistivity is given by

$$
\left.\rho=\rho_{0} \exp \left\{D / L_{0}\left[\gamma C_{m}\right)^{-1 / 3}-1\right]\right\},
$$

where $\gamma$ depends on the grain shape, and $\rho_{0} \sim 10^{-3} \Omega \mathrm{cm}$. 

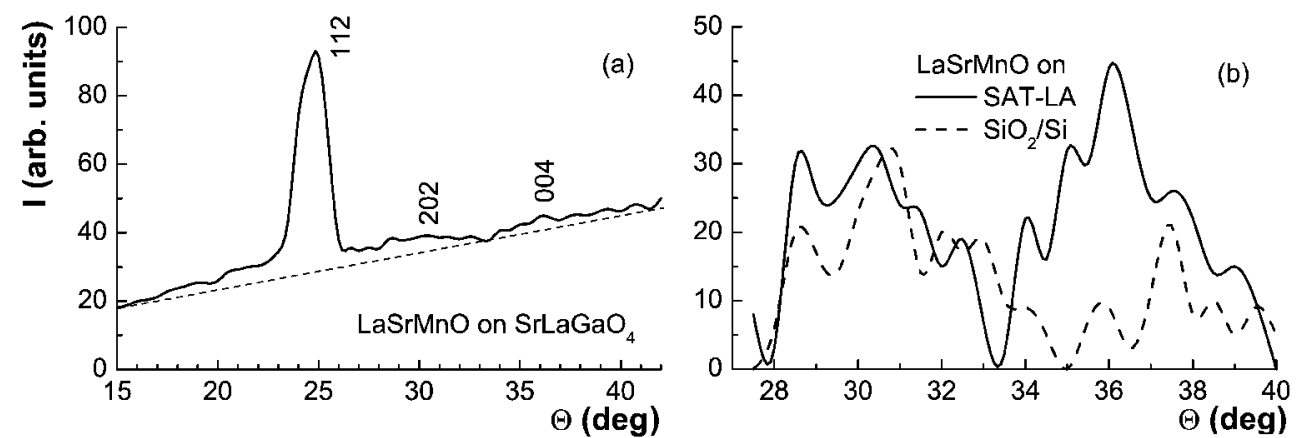

Fig. 1. Examples of diffractograms obtained for the investigated films. (a) Full diffractogram of X-ray ( $\mathrm{Cr} K_{\alpha}$ ) scattering on LaSrMnO film grown on $\mathrm{SrLaGaO}_{4}$ at $T_{\mathrm{s}}=700^{\circ} \mathrm{C}$. (b) Diffractogram fragments in the region of diffusive maxima due to $\mathrm{Mn}-\mathrm{O}$ clusters in crystalline LaCaMnO films grown on different substrates: $1-\mathrm{SiO}_{2} / \mathrm{Si}$, $2-\left(\mathrm{SrAl}_{0.5} \mathrm{Ta}_{0.5} \mathrm{O}_{3}\right)_{0.7}\left(\mathrm{LaAlO}_{3}\right)_{0.3}$, at the same temperature $T_{\mathrm{s}}=725^{\circ} \mathrm{C}$.

We have selected two groups of samples differing by their $R(T)$ dependence. In the first group, the resistivity for $T<T_{\max }$ changed slowly, $(\mathrm{d} R / \mathrm{d} T) / R$ $<0.7 \% / \mathrm{K}$ (Figs. 2 and 3 ). For the samples in this group, simultaneous plots of resistivity and magnetization versus temperature reveal that the maximum of $R(T)$ is located on the slope of the $M(T)$ curve, as if the electronic subsystem has a delayed reaction to the changes in the magnetic subsystem, and the latter has to reach some threshold state in order to induce changes in the electronic properties.

The samples in the second group exhibit rapid decrease in the resistivity in a narrow temperature interval with the rate of change $(\mathrm{d} R / \mathrm{d} T) / R \approx 4 \% / \mathrm{K}$, i.e. 5-6 times higher than in the first group. The $\sigma$ magnitude of cooled samples $\left(10^{3}-10^{4} \Omega^{-1} \mathrm{~cm}^{-1}\right)$ exceeds $\sigma_{\min }$, and - in contrast to the samples of the first

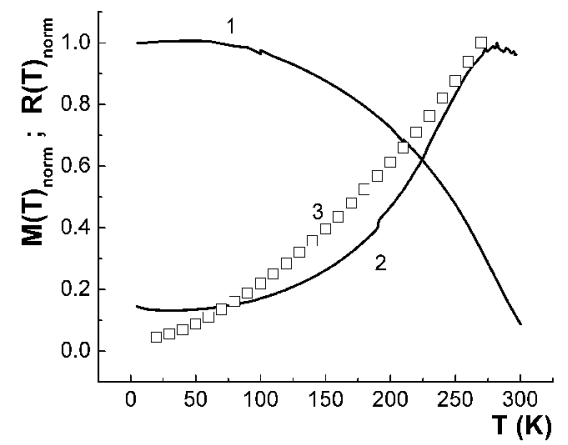

Fig. 2. Temperature dependence of magnetization (1) and resistivity (2) for LaSrMnO film grown on $\mathrm{SrLaGaO}_{4}$ substrate at $T_{\mathrm{s}}=650^{\circ} \mathrm{C}$. (3) - theoretical fit of curve (2) obtained for $C_{m}^{(0)}=0.1, T_{\max }=270 \mathrm{~K}, B=1 \times 10^{-3} \mathrm{~K}^{-1}$. 


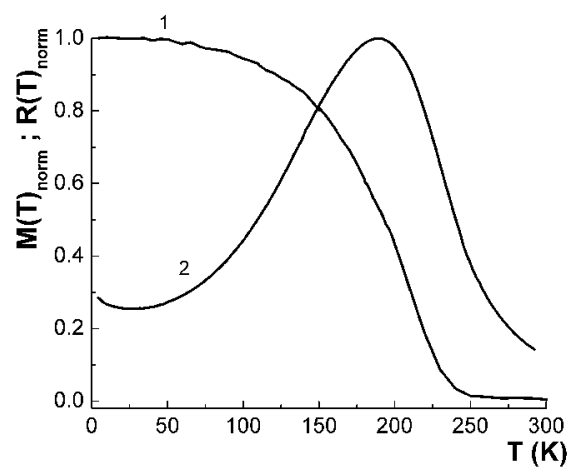

Fig. 3. Temperature dependence of magnetization (1) and resistivity (2) for LaCaMnO film grown on $\mathrm{SiO}_{2} / \mathrm{Si}$ substrate at $T_{\mathrm{s}}=725^{\circ} \mathrm{C}$.

group - the conductance for $T<230 \mathrm{~K}$ has a metallic character. At low temperatures, the $R(T)$ curve can be approximated by the linear, characteristic for metals, dependence $R(T)=R_{0}(1+\alpha T)$, where $\alpha=7.1 \times 10^{-3} \mathrm{~K}^{-1} \div 3.7 \times 10^{-3} \mathrm{~K}^{-1}$, i.e. $\alpha$ values are close to those exhibited by pure metals (e.g. Co and $\mathrm{Al}$ ). In this group, minimum in $R(T)$ is absent, the maximum coincides with the beginning of magnetization $M(T)$ increase, and maximal $\mathrm{d} R / \mathrm{d} T$ and $\mathrm{d} M / \mathrm{d} T$ rates (of opposite signs) are observed at the same temperatures.

Comparison of the samples of the two groups shows that the position of $R(T)$ curves with respect to $M(T)$ is neither directly related to the Curie temperature nor to the magnitude of saturation magnetization. Different threshold values of $M(T) / M(5 \mathrm{~K})$ reflect different sensitivities of the electronic subsystem to the changes in the magnetic subsystem.

Diffractograms of the investigated films exhibit a bright maximum $\left(\theta_{\mathrm{Cr}} \approx 25^{\circ}\right)$, due to the basic group of planes in the crystalline film, as well as weak diffusive maxima $\left(\theta_{\mathrm{Cr}} \sim 27 \div 38^{\circ}\right)$ due to the clusters (Fig. 1$)$. In the first group of samples, the (202) planes (the layers parallel to the $y$ axis) are dominating, and the dominance of these planes in the cluster structure is reflected by the reduction of $\sigma$. In the second group (films on $\left.\left(\mathrm{SrAl}_{0.5} \mathrm{Ta}_{0.5} \mathrm{O}_{3}\right)_{0.7}\left(\mathrm{LaAlO}_{3}\right)_{0.3}\right)$, the intensity of diffusive scattering is distributed symmetrically with respect to the center. The vertices of the diffraction complexes correspond to the fragments of (202) and (004) planes of the orthorhombic phase. Each of the two complexes yields information on the structural fractions comprising clusters with variable layer separation, both compressed and expanded.

The fundamental difference between (004) and (202) clusters is that the (004) planes are more closely spaced $\left(d_{004}=1.94377 \AA\right)$ than the $(202)$ ones $\left(d_{202}=2.25689 \AA\right)$. This is related to the variable length of $\mathrm{Mn}-\mathrm{O}$ bonds for different $\mathrm{Mn}$ ionization states. In (004) clusters dominate $\mathrm{Mn}^{3+}$ and $\mathrm{Mn}^{4+}$ ions with shorter bonds, while (202) clusters are mostly composed from $\mathrm{Mn}^{2+}$ and $\mathrm{Mn}^{3+}$ 
ions forming longer bonds with oxygen. Metallic conductance and ferromagnetism are realized in clusters composed of fragments of layers with small $d$, containing $\mathrm{Mn}^{3+}$ and $\mathrm{Mn}^{4+}$ ions in comparable quantities.

The above results indicate a variety of mechanisms which induce the neighboring atomic clusters into structural liaisons increasing the volume of the metallic phase and thus facilitate the magnetic ordering and lead to increased conductivity of the films. Atomic order appears to determine the interrelationship between the electronic and magnetic subsystems.

The dielectric-metal transition mechanism as well as the $\mathrm{d} M / \mathrm{d} T$ and $\mathrm{d} R / \mathrm{d} T$ rates is determined by the pace of cluster structure reorganization. This process is the easiest for films grown on $\left(\mathrm{SrAl}_{0.5} \mathrm{Ta}_{0.5} \mathrm{O}_{3}\right)_{0.7}\left(\mathrm{LaAlO}_{3}\right)_{0.3}$, as their cluster structure is represented by groups involving highly ionized $\mathrm{Mn}^{3+}$ and $\mathrm{Mn}^{4+}$ manganese states with dominating ferromagnetic interactions. It is not accidental that in these samples $R(T)$ maximum coincides with the beginning of magnetization increase (Fig. 4). In the first group of samples characterized by mixed cluster structure and by competing ferro- and antiferromagnetic interactions, the transition into magnetically ordered state is smeared, $R(T)$ maximum is shifted below the Curie temperature, and the transition dielectric-metal is obscured.

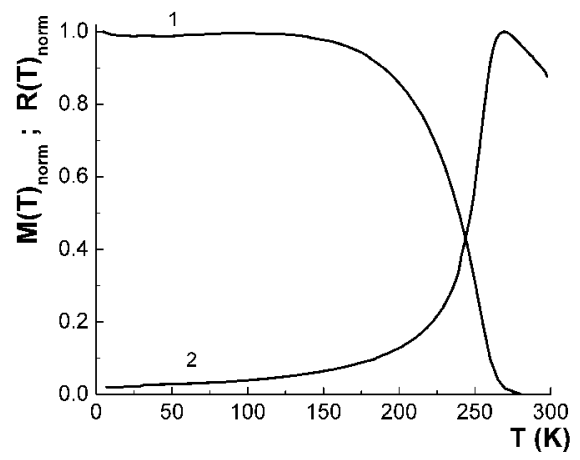

Fig. 4. Temperature dependence of magnetization (1) and resistivity (2) for LaCaMnO film grown on $\left(\mathrm{SrAl}_{0.5} \mathrm{Ta}_{0.5} \mathrm{O}_{3}\right)_{0.7}\left(\mathrm{LaAlO}_{3}\right)_{0.3}$ substrate at $T_{\mathrm{s}}=725^{\circ} \mathrm{C}$.

It can be shown [12] that if the conduction mechanism relies on tunneling between clusters, then the film resistivity in the interval $T_{\min }<T<T_{\max }$ is given by the expression

$$
\rho=\rho_{0} \exp \left\{\frac{D^{(0)}}{L_{0}} \frac{\left[(\gamma X)^{-1 / 3}\left(1+\frac{1}{3} \frac{B}{X} T\right)-1\right]}{|Y|^{\nu}\left|1-\frac{B}{Y} T\right|^{\nu}}\right\},
$$

where $X=C_{m}^{(0)}+B T_{\max }, Y=C_{m}^{(0)}+B T_{\max }-C_{m}^{c r i t}, C_{m}^{\text {crit }}=0.5$, and $\nu=0.85$ is the critical index. In accordance to the experimental data, we can assume in this model that $D$ changes as the correlation radius in the percolation theory: $D(T) \approx D^{(0)}\left|\left[C_{m}^{(0)}+B\left(T_{\max }-T\right)\right]-C_{m}^{\mathrm{crit}}\right|^{-\nu}$ and on cooling the sample below 
the Curie temperature, the concentration of metallic phase $C_{m}$ depends linearly on temperature, $C_{m}=C_{m}^{(0)}+B\left(T_{\max }-T\right)$. In deriving Eq. (3) it is also assumed that $B T / X<1$. Increased size of FM clusters in the AFM matrix is revealed on the $M(T)$ characteristic as a reduction of the length of $M=$ const section with increasing magnetic field or as the total absence of this section for $H \geq 1 \mathrm{kOe}$ [13]. From Eq. (3) it is seen that in the considered model the resistivity decreases with temperature. As a test of the validity of (3), we have calculated the $\rho(T)$ curve for $C_{m}=0.1$ and plotted it in Fig. 2 together with experimental data for a sample which in optical measurements exhibited $C_{m}=0.085$. As seen, the agreement between calculated and measured data is satisfactory.

\section{Acknowledgment}

This work was supported by grant PBZ-KBN-013/T08/19 of the State Committee for Scientific Research. The authors thank H. Bielska-Lewandowska and M. Tanecka for their help with transport measurements.

\section{References}

[1] J.M.D. Coey, M. Viret, S. von Molnar, Adv. Phys. 48, 167 (1999).

[2] M.B. Salamon, M. Jaime, Rev. Mod. Phys. 73, 583 (2001).

[3] S.V. Trukhanov, I.O. Troyanchuk, N.V. Pushkarev, H. Szymczak, JETP 95, 308 (2002)

[4] H.L. Ju, Hyunchul Sohn, J. Magn. Magn. Mater. 167, 200 (1997).

[5] S.H. Chun, Y. Lyanda-Geller, M.B. Salamon, R. Suryanarayanan, G. Dhalenne, A. Revcolevschi, J. Appl. Phys. 90, 6307 (2001).

[6] N.G. Bebenin, R.I. Zanullina, V.V. Mashkautsan, V.S. Gaviko, V.V. Ustinov, Ya.M. Mukovski, D.A. Shulyatev, JETP 90, 1027 (2000).

[7] Guo-meng Zhao, V. Smolyaninova, W. Prellier, H. Keller, Phys. Rev. Lett. 84, $6086(2000)$.

[8] V.D. Okunev, Z.A. Samoilenko, V.M. Svistunov, A. Abal'oshev, E. Dinowska, P. Gierlowski, A. Klimov, S.J. Lewandowski, J. Appl. Phys. 85, 7282 (1999).

[9] Z.A. Samolenko, V.D. Okunev, E.I. Pushenko, T.A. D'yachenko, A. Cherenkov, P. Gierlowski, S.J. Lewandowski, A. Abal'oshev, A. Klimov, A. Szewczyk, Techn. Phys. 48, 250 (2003).

[10] N.F. Mott, Metal-Insulator Transitions, Taylor \& Francis, London 1990.

[11] A.B. Khanikaev, A.B. Granovski, J.P. Clerc, Phys. Solid State 44, 1611 (2002).

[12] V.D. Okunev, Z.A. Samoilenko, A. Abal'oshev, M. Berkowski, P. Gierlowski, S.J. Lewandowski, A. Szewczyk, H. Szymczak. R. Szymczak, to be published.

[13] V.D. Okunev, Z.A. Samoilenko, T.A. D'yachenko, R. Szymczak, S.J. Lewandowski, H. Szymczak, M. Baran, P. Gierlowski, Phys. Solid State, in print 2003. 